\title{
Front Structures in a Real Ginzburg-Landau Equation Coupled to a Mean Field
}

\author{
Henar Herrero \\ Departamento de Física y Matemática Aplicada, Facultad de Ciencias, \\ Universidad de Navarra, 31080 Pamplona, Navarra, Spain \\ Hermann Riecke \\ Department of Engineering Sciences and Applied Mathematics \\ Northwestern University, Evanston, IL 60208, USA
}

November 20, 2018

\begin{abstract}
Localized traveling wave trains or pulses have been observed in various experiments in binary mixture convection. For strongly negative separation ratio, these pulse structures can be described as two interacting fronts of opposite orientation. An analytical study of the front solutions in a real Ginzburg-Landau equation coupled to a mean field is presented here as a first approach to the pulse solution. The additional mean field becomes important when the mass diffusion in the mixture is small as is the case in liquids. Within this framework it can lead to a hysteretic transition between slow and fast fronts when the Rayleigh number is changed.
\end{abstract}

PACS numbers: 47.20.Ky, 03.40.Kf, 47.25.Qv

To appear in: J. Bif. and Chaos 


\section{Introduction}

Convection in binary mixtures occurs through a Hopf bifurcation for sufficiently strong coupling between the thermal and the concentration field. This bifurcation is usually subcritical and leads to traveling waves. Strikingly, they can form localized-wave trains (LTW). Two classes of LTW seem to exist: a discrete set of relatively short, pulselike structures, which resemble solitons in the nonlinear Schrödinger equation, and longer LTW, which resemble a pair of fronts connecting the convective and the conductive state. Previously, it seemed that the latter have arbitrary length. Very recent experiments showed, however, that this is not the case and that their stable length is fixed, too [Kolodner, 1993]. In both cases the drift velocity is very small. Theoretical efforts to understand the LTW within the framework of reduced equations like the complex Ginzburg-Landau equation (CGL) have focussed on different points of view: 1) considering the CGL as a perturbation of the nonlinear Schrödinger equation, one can show the stability of short LTW [Thual \& Fauve, 1988, Fauve \& Thual, 1990]; 2) in the weakly dispersive limit, i.e. when the imaginary parts of all of the coefficients in the CGL are very small, bound pairs of fronts can be stable with their distance diverging when the imaginary parts go to zero [Hakim et al., 1990, Hakim \& Pomeau, 1991]. In both approaches the discrepancy between the calculated linear group velocity $s$ and the considerably smaller observed velocity $v$ of the LTW requires that nonlinear contributions balance the linear group velocity over a range of parameters.

Taking into account full numerical simulations of the Navier-Stokes equations, which show the relevance of a large-scale concentration field [Barten et al., 1991], it has been suggested [Riecke, 1992] that the smallness of the drift velocity $v$ of the LTW is due to an additional slow time scale in the binary-mixture system. It arises due to the smallness of the Lewis number $\mathcal{L}$, which measures the ratio of molecular to thermal diffusion, and leads to an additional dynamical degree of freedom - beyond the convective amplitude, which corresponds to a concentration mode. Numerical simulations of the resulting coupled PDE show that for small $\mathcal{L}$ the pulse velocity is drastically reduced, and the effect of the group velocity $s$ on the pulse velocity is strongly diminished as compared to the conventional CGL.

In both cases described above [Fauve \& Thual, 1990,Hakim \& Pomeau, 1990] the localization mechanism is due to the dispersion of the waves, i.e. no localization would be possible if the imaginary coefficients in the CGL vanished identically. The strong effect of the concentration mode on the velocity of the LTW raises the question whether localization could arise solely due to the concentration mode without any dispersion. Here we make a first step towards answering this question. We investigate analytically single front solutions in the presence of this mean field and focus on the dispersionless case.

The equations proposed in [Riecke, 1992] are

$$
\begin{aligned}
\partial_{T} A+s \partial_{X} A & =d \partial_{X}^{2} A+(a+f C) A+c A|A|^{2}+p A|A|^{4} \\
\partial_{T} C & =\delta \partial_{X}^{2} C-\alpha C+h \partial_{X}|A|^{2} .
\end{aligned}
$$


They represent a minimal model for the mechanism of interest. The complete equations up to cubic order, including the values of the coefficients for free-slip-permeable boundary conditions, are given in [Riecke, 1992]. Without dispersion, the real Ginzburg-Landau equation for the real amplitude, without coupling to the $C$-field and after a rescaling can be written as

$$
\partial_{t} A+s \partial_{x} A=\partial_{x}^{2} A-\frac{\partial \mathcal{V}}{\partial A}
$$

with

$$
\mathcal{V}(A)=\frac{1}{2} A^{2}-\frac{c}{4} A^{4}+\frac{1}{6} A^{6} .
$$

In the subcritical case $c \geq 0$ the potential $\mathcal{V}$ has two relative minima for $A \geq 0$, one at $A=0$ and

another at $A=A^{*}$. At $c=c_{c}\left(\equiv \frac{4}{\sqrt{3}}\right)$ the potential has the same value for the conductive state $A=0$ and for the convective state $A=A^{*}$, and the front solution connecting the conductive state to the convective state is stationary. For $c>c_{c}$ the $A=A^{*}$ state invades the trivial state. Due to the attractive interaction between the fronts, domains of definite size exist, they are, however, unstable. The size of these domains diverges when $c$ tends to $c_{c}$ and in this limit the localized unstable domain can be described perturbatively as a bound state of two fronts.

\section{Front Solutions}

The front between the trivial state and the convective state for $C=0$ is at rest for $c=c_{c}$ and has the form

$$
A_{L}\left(x-x_{0}\right)=A^{*}\left(\frac{1}{2}\left(1+\tanh \left(\frac{x-x_{0}(t)}{\xi}\right)\right)\right)^{1 / 2}
$$

where $A^{* 2}=\sqrt{3}$ and $\xi=1$. If $c \neq c_{c}$ and $C \neq 0$ the front starts to move. In a frame moving with velocity $v$, in which the front is stationary, Eqs.(11)-(2) read

$$
\begin{aligned}
(s-v) A^{\prime} & =A^{\prime \prime}-A+c A^{3}-A^{5}+C A \\
-v C^{\prime} & =\delta C^{\prime \prime}-\alpha C+h \partial_{x} A^{2} .
\end{aligned}
$$

Here $f$ has been absorbed into $h$ and $C$. Assuming $c_{1} \equiv c-c_{c}$ and $C$ small, $A_{L}$ is a zeroth order approximation to the moving front,

$$
A=A_{L}+\epsilon A_{1}+\ldots \quad C=\epsilon C_{1}+\ldots
$$

and $s-v=O(\epsilon)$. In order to solve the inhomogeneous equation for $C$ we assume strong advection and expand $C$ in $v^{-1}$. In this limit Eq.(可) becomes a first-order equation and only the boundary condition on $C$ ahead of the front can be satisfied. Thus, in the case $v>0(v<0)$ one has $C \rightarrow 0$ for $x \rightarrow \infty(x \rightarrow-\infty)$. In order to satisfy the other boundary condition as well one has to take into account that in back of the front, where $\partial_{x} A^{2}$ goes to zero, the term 
$\alpha C / v$ becomes dominant. This can be done using a suitable matching procedure. To obtain the velocity of a single front this is, however, not necessary. Thus, one obtains

$$
\begin{aligned}
C & =-\frac{h}{v} A_{L}^{2}+\frac{h \delta}{v^{2}}\left(A_{L}^{2}\right)^{\prime}-\frac{h \alpha}{v^{2}} \int_{-\infty}^{x} A_{L}^{2} d x+O\left(\frac{1}{v^{3}}\right), \quad v<0, \\
C & =-\frac{h}{v}\left(A_{L}^{2}-A^{* 2}\right)+\frac{h \delta}{v^{2}}\left(A_{L}^{2}\right)^{\prime}+\frac{h \alpha}{v^{2}} \int_{x}^{\infty}\left(A_{L}^{2}-A^{* 2}\right) d x+O\left(\frac{1}{v^{3}}\right), \quad v>0,
\end{aligned}
$$

This expansion is inserted into the equation for $A$. The leading order equation is solved by $A_{L}$. At $O(\epsilon)$ a solvability condition arises due to the translational symmetry of the system. Projection onto the left zero-eigenvector $\partial_{x} A_{L}$ yields an equation for the velocity $v$,

$$
s=v+\sqrt{3}\left(c_{1}+\frac{h}{v}\right)+\frac{2 \sqrt{3} \delta h}{3 v^{2}}-\frac{\sqrt{3} \alpha h}{v^{2}} .
$$

Thus, for $h>0$ the absolute value of the velocity $(|s|<|v|)$ of a leading front $(v<0)$ is increased by the concentration field at leading order, whereas that of a trailing front $(v>0)$ is decreased. Considering that positive values of $C$ increase the local growth rate of $A$, this is expected and illustrated in fig.1. There, two typical numerical solutions of Eqs.(11)-(2) are shown for $v<0$ and for $v>0$. In both cases the positive concentration mode supports the growth of the convective state.

Eq.(11) suggests a hysteretic transition from fast fronts to slow fronts when the group velocity $s$ is decreased with the latter traveling opposite to the group velocity. Although this transition occurs outside of the regime of validity of Eq.(11), this is in fact found in numerical simulations of Eqs.(11)-(2) [Herrero \& Riecke, in preparation] and is reminiscent of a similar transition for pulses [Riecke, 1993]. Experimentally of interest should be the fact that this transition in the front velocity can also be achieved by changing $c_{1}$. Due to our scaling of Eqs.(11)-(2) this is equivalent to changing the Rayleigh number. Thus, the velocity of a single front is expected to be strongly nonlinear in the Rayleigh number whereas without the concentration mode it would be linear to lowest order.

In conclusion, we have investigated the influence of an additional mean field on the dynamics of propagating fronts in a real Ginzburg-Landau equation without dispersion. This is considered a first step towards the understanding of localized traveling-wave pulses which have been found in simulations of these equations and which appear to be similar to localized waves found in binary-mixture convection at strongly negative separation ratio. To treat the interaction of such fronts one has to take into account the slow decay of the concentration mode behind the front. In a limiting case in which the fronts are very steep this decay can be seen to be the dominant contribution to the interaction and can lead to stable bound states of two fronts, i.e. stable localized waves [Herrero \& Riecke, in preparation].

\section{Acknowledgements}

H.H. was supported by a grant of the F.P.I. program Ref.AP90 09297081 (M.E.C.). H.R. gratefully acknowledges the hospitality of the University of Navarra. The work of both authors 
was supported by DOE through grant DE-FG02-92ER14303. We gratefully acknowledge also discussions with W.L.Kath. 


\section{References}

[1] Barten, W., Lüecke, M. \& Kamps, M. [1991] "Localized Traveling-Wave Convection in Binary Fluid Mixture", Phys. Rev. Lett. 66, 2621.

[2] Barten, W., Lüecke, M. \& Kamps, M. [1992] "Convection in Binary Mixtures: the Role of the Concentration Field", Physica D.

[3] Fauve, S. \& Thual, O. [1990] "Solitary Waves Generated by Subcritical Instabilities in Dissipative Systems", Phys. Rev. Lett. 64, 282.

[4] Hakim, V., Jakobsen, P. \& Pomeau, Y. [1990] "Fronts vs Solitary Waves in Nonequilibrium Systems", Europhys. Lett. 11, 19.

[5] Hakim, V. \& Pomeau, Y. [1991] "On Stable Localized Structures and Subcritical Instabilities", Eur. J. Mech. B10, No. 2 Suppl., 137-143.

[6] Herrero, H. \& Riecke, H.(in preparation).

[7] Kolodner, P. [1991] "Drifting Pulses of Traveling-Wave Convection", Phys. Rev. Lett. 66, 1165-1168.

[8] Kolodner, P. [1993], preprint.

[9] Riecke, H. [1992] "Self-Trapping-Wave Pulses in Binary Mixture Convection", Phys. Rev. Lett. 68, 301-304.

[10] Riecke, H. [1992] "Ginzburg-Landau Equation Coupled to a Concentration Field in BinaryMixture Convection", Physica D 61, 253.

[11] Riecke, H. [1992] "Self-Trapping of Traveling-Wave Pulses" in Spatio-Temporal Organization in Nonequilibrium Systems, eds. S.C. Müller \& T. Plesser, Projekt Verlag, 222-223.

[12] Thual, O. \& Fauve, S. [1988] "Localized Structures Generated by Subcritical Instabilities", J. Phys. (Paris) 49, 1829. 


\section{Figure Captions}

Figure 1: Typical front for $c=-2.309, \delta=0.009, \alpha=0.01$ and $h=0.03$.

a) $s=0.15$, b) $s=0.5$. 


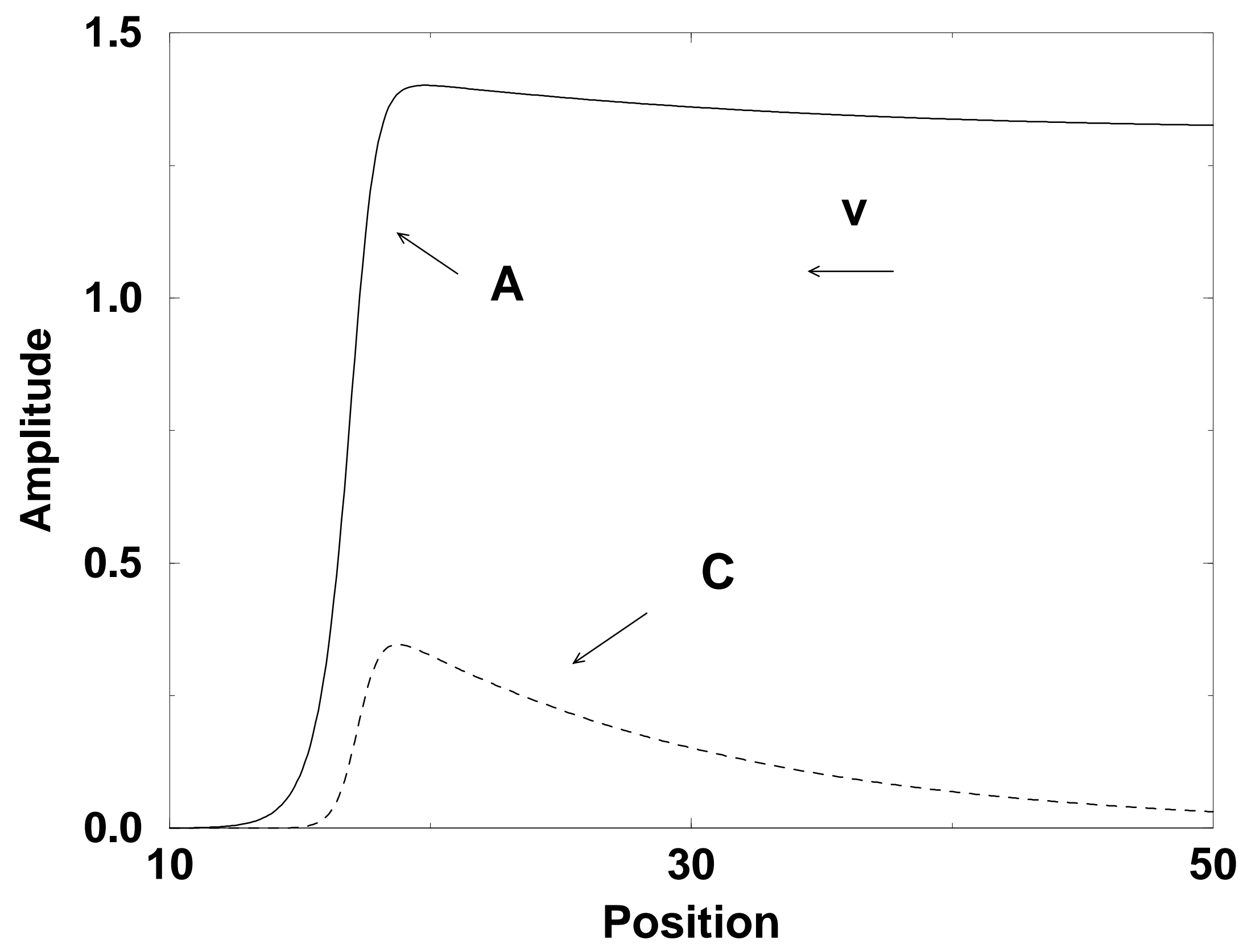




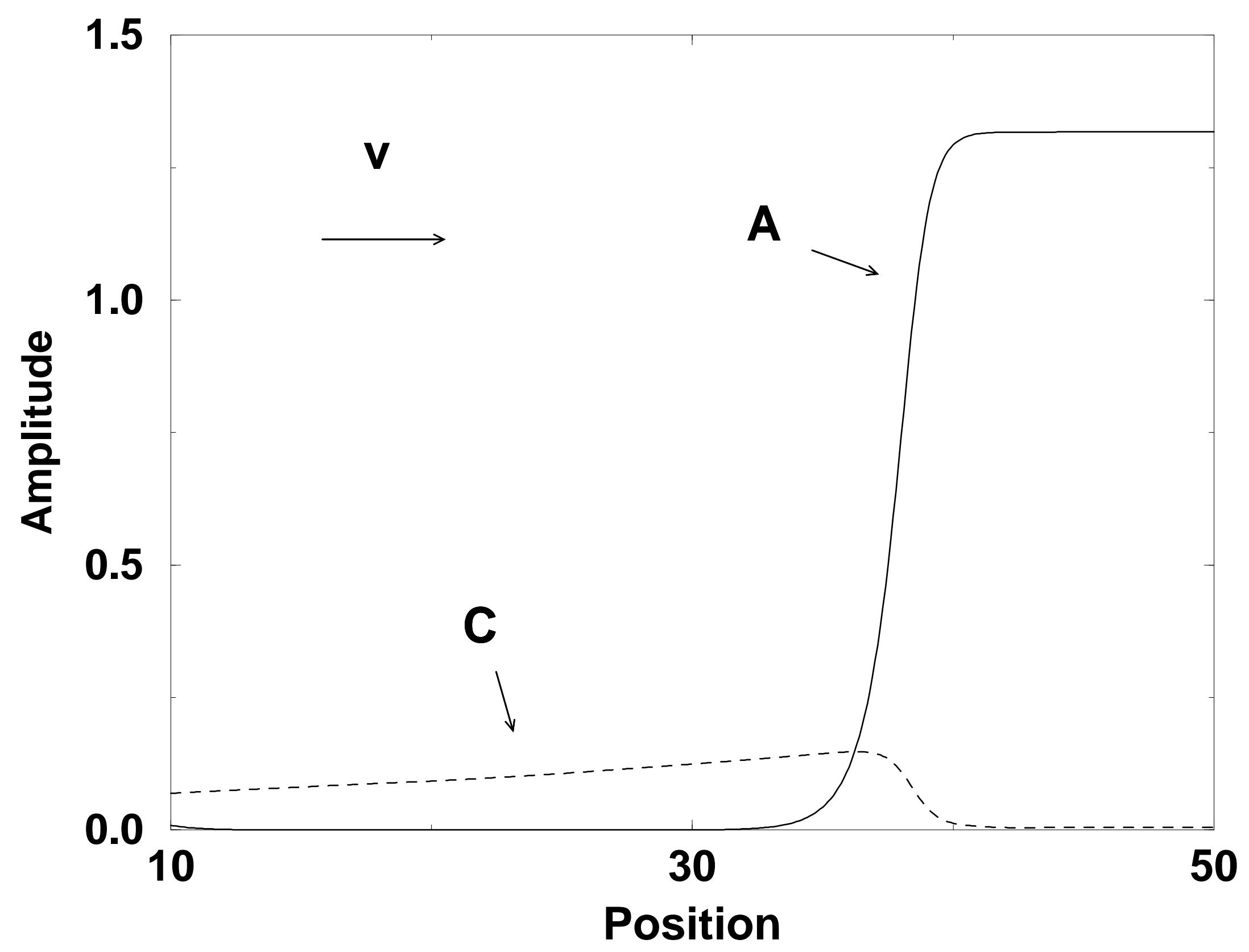

